\title{
Cinco Casos de Coma Eclamptico Tratados con Cortisona
}

\author{
Doctor. Eduardo Acosta Bendek \\ De la Sociedad de Obstetricia y Ginecologia del Atlántico. \\ Trabajo elaborado en el Servicio de Maternidad "Leopoldina de \\ Santos" del Hospital de Barranquilla y leído en la sesión ordinaria del \\ mes de abril en la Sociedad de Obstetricia y Ginecologia del Atlántico.
}

Desde que se describió el cuadro clásico de la intoxicación gravídica hasta nuestros días, continuamos en la misma obscuridad en lo que se relaciona con su etiopatogenia y tratamiento, hasta el extremo de que bien podría aplicársele la hermosa y significativa frase de Winston Churchill y decir con él que se está frente a "una adivinanza dentro de un misterio, envuelto en un enigma". Sin embargo, parece disiparse en parte el obscuro panorama actual a este res. pecto, en la nueva concepción creada por SELYE en los últimos años denominada "Síndrome General de Adaptación", dentro de la cual puede situarse la Eclampsia. Sindrome que me voy a permitir exponer muy brevemente con el fin de ser más explícito en este escrito.

Como bien es sabido, todo agente causante de reacciones morbosas produce a su vez, en el organismo dos clases de efectos: uno específico, que está intimamente ligado al agente patógeno, y que produce reacciones específicas propias de cada enfermedad, por ejemplo, la lesión chancrosa, en la primoinfección sifilitica, la supuración peritoneal, en la peritonitis aguda, etc., y las reacciones inespecificas comunes a muchas enfermedades, que se presentan proporcionalmente de acuerdo al grado de lesión que produzca el agente causante de las mismas y que vienen a mezclarse con las reacciones específicas. Estos fenómenos generales e inespecificos son los que vienen a formar el "Síndrome General de Adaptacion", el cual requicre para producirse, según el autor citado, la presencia de un "alarming stimulus" "alarmógeno" al decir del pro fesor Rodolfo Pascualini, quien lo define citando a SELYE, en los términos siguientes: "Ciralquicr agente capaz de provecar primero una reacción de alar ma y, si su accion se prolonga, el "Sindrome Cenerat de Ada "ación" completo, sindrome que croluciona en las tres fasces siguicntes: 1:-Recición de alarma. 
$2^{i 1}$-Estado de resistencia y $3^{a}$-Estado de agotamiento, que SELYE explica de la siguiente manera:

$l^{\text {a }}$-Reacción de alarma que es definida como "la suma de todos los fenó menos no específicos provocados por la exposición brusca a estímulos que afectan grandes extensiones del organismo y a los cuales éste no está adaptado cualitativa y cuantitativamente".

2a-Estado de resistencia, que "representa la suma de todas las reacciones generales no específicas, provocadas por la exposición prolongada a estímulos a los cuales el organismo ha adquirido adaptación".

3:-Estado de agotamiento, que "representa la suma de todas las reacciones generales no específicas que se desarrollan finalmente como resultado de la sobre exposición a estímulos frente a los cuales se desarrolló adaptación que ulteriormente no pudo ser sostenida".

Como es obvio suponer, estos cambios representan un gran esfuerzo por parte del organismo para sobreponerse a las exigencias producidas por el agente agresor, esfuerzo que puede ser normal, exagerado o mínimo, por otra parte SELYE y otros investigadores ( ${ }^{1}$ ) han estudiado en los últimos años los efectos de las hormonas cortico-adrenales en el desarrollo de la resistencia orgánica frente a influencias dañinas para el organismo, que actúan durante períodos prolongados y han observado al comienzo una gran secreción de hormonas corticales, seguida por hipertrofia y aumento de actividad de la corteza suprarrenal.

Este nuevo concepto de "enfermedades de adaptación" parece proporcionarnos alguna luz sobre el intrincado problema de la intoxicación gravídica, como lo veremos en el desarrollo de este trabajo.

Basado en esta nueva concepción y en las experiencias verificadas con éxito por algunos obstetras sobre el tratamiento de la eclampsia con la CORTISONA, me indujeron a ensayar dicho compuesto en esta grave complicación de la mujer embarazada. Así fue como en colaboración con mis compañeros de trabajo, de la Maternidad "Leopoldina de Santos" del Hospital de Barranquilla, hicimos el tratamiento de cinco casos graves de coma eclámptico, en los cuales había fracasado el tratamiento corriente, y por cuyos halagüeños resultados me he permitido publicarlos en forma de una comunicación previa. A continuación doy lectura a las cinco historias clínicas, de cada una de las cuales he elaborado un extracto.

\section{CASOS REPORTADOS:}

Caso No 1. Paciente: C. G. de 16 años de edad, primigestante. Llega al servicio de Maternidad "Leopoldina de Santos", a las 4 y media a. m. del día 9 de diciembre de 1953, con embarazo de 8 meses, en franco trabajo de parto y con ruidos fetales negativos, quien es presa de un ataque convulsivo en la mesa de trabajo pocos momentos después de su llegada al Servicio, seguido de un estado de coma la presión arterial maxima ce de loo y la minima de 120, pulso 90 por minuto, respiraciones 30 por minute, we mara $37^{\circ}$ (.) Se sondea y re retiran 100 c.c. de orina completamente hematurica que se 
cnvían al laboratorio. El parto se inició a las 11 p. m. del día 8 y terminó a las 5 y media a. m. del día siguiente, con un niño muerto no macerado $y$ prematuro. Inmediatamente después del primer ataque convulsivo se le apli (c) el tratamiento corriente, a base de barbitúricos. Sulfato de Magnesio al 25\% cada 4 horas, intramuscular, solución dextrosada al 50\%, 40 c.c. cadi 4 horas por vía intravenosia, más vitamina C. a la dosis de un gramo. El estado comatoso de la enferma es interrumpido por dos ataques conrulsivos más. uno a las 7 horas y el otwo a las 12 horas después del parto. El estado de la enferma se hace más serio, y se procede a aplicar CORTISONA a las 6 p. m., a razón de $100 \mathrm{mg}$. cada 8 horas por vía intramuscular. Ocho horas después de la aplicación del medicamento la enferma tiene una franca mejoría, la diuresis se hace presente, el estado de coma comienza a ceder, la respiración se hace más normal, la presión arterial es de $140 \times 80$, pulso 100 por minuto. A las 24 horas de haber comenzado el tratamiento la enferma se encontraba en estado de lucidez mental, la diuresis había aumentado, la presión arterial había descendido a $110 \times 70$, pulso 98 , respiraciones 20 por minuto. A las 48 horas la enferma estaba restablecida y el estado general era muy satisfactorio. Sólo se aplicaron en total $500 \mathrm{mg}$. de CORTISONA. Esta enférma hizo más tarde un cuadro tifoso, que se comprobó por el laboratorio. La enferma sale del Servicio el día 10 de enero siguiente, en franco estado de salud.

Exámenes de Laboratorio: Orina $5 \mathrm{gms}$. de albúmina, cilindros granulosos 2 cruces, hialinos 2 cruces, hematíes 3 cruces. Azohemia $96 \mathrm{mg}$. \%. Hemo grama: eritrocitos 3.440.000; leucocitos 14.650; Hg. 70\%; Glicemia $80 \mathrm{mg}$. \%; Fórmula blanca: polinucleares, $89 \%$, neotrófilos, eosinófilos y basófilos 0 , linfocitos 10, monocitos 1. Reacción de Widal positivo para el tífico $\mathrm{H}$ y $\mathrm{O}$.

Caso No 2. Paciente: I. T. de 23 años de edad, primigestante, de raza negra, embarazo de 8 y medio meses, llega al Servicio de Maternidad, el 14 de febrero del presente año, a la una y media de la madrugada, en estado de coma y agitación, después de dos ataques convulsivos. Tensión arterial : Mx. de 130 y Mn. de 110, pulso filiforme e incontable, respiración estertorosa, 34 por minuto, temperatura 37 y medio grados $C$., y casi en estado de anasarca. Se son dea y se retiran 60 c.c. de orina que se envían al laboratorio. Se hace aplica ción inmediata del tratamiento corriente, A las $7 \mathrm{a} . \mathrm{m}$. el estado do la enferm. se hace más desesverante, la tensión arterial máxima desciende a 140 y la mínima no se pudo registrar. Ruidos fetales negativos. Se aplican analépticos y se comienza la administración de la CORTISONA, a dosis de $100 \mathrm{mg}$. in tramuscular cada 8 horas. Una hora más tarde la enferma comienza a reaccionar favorablemente, la respiración se hace menos estertorosa, la tensión ar terial máxima es de 160 y la mínima 120. la frecuencia del pulues es de 116 dé bil y rímico $\mathrm{Al}$ cumplirse las t horas de haberse aplicado el medicamento, el cstado comatoso de la enforma comienza a ceder, la respiración menos ester torosa entrando a su ritmo normal. La tension arterial y pulso en el mismo estado que de equistado anteriomente. A las 6 a. m. det dia signiente o sea a las 23 hora de comenzado el tratamiento, la enferma cntra en estado de la 
cidez mental, la tensión arterial máxima 160 y mínima 100, pulso 100 por minuto, rítmico, respiración normal. El trabajo de parto se inició a las 5 y media a. $\mathrm{m}$. del día 15 y terminó a las $10 \mathrm{a} . \mathrm{m}$. del mismo día, fue rápido y espontáneo. Con niño muerto y sin comienzo de maceración, y presentó una circular del cuello. La dosis total de CORTISONA fue de $500 \mathrm{mg}$.

Exámenes de Laboratorio: Orina, albúmina 2 gms., cilindros hialinos: + +; gránulos: +; hematíes: +. Glicemia $110 \mathrm{mg}$. \%; azohemia $32 \mathrm{mg}$. \%. Kahn negativo. Fondo de Ojo: ojo derecho edema papilar con hemorragias peripapilares, congestión venosa y espasmo arterial, cruces positivos y exudados abundantes. En el ojo izquierdo, el edema de la papila estaba más pronunciado, lo demás exactamente igual al anterior.

Caso No 3. Paciente: M. M. de 25 años de edad, de color, multigestante, llega al Servicio de Maternidad a las 4 y media de la mañana del día 4 de noviembre de 1953, en un franco ataque convulsivo seguido de un estado de coma. Embarazo a término, ruidos fetales negativos, tensión arterial Mx. y Mn. 0, temperatura $38^{\circ} \mathrm{C}$., pulso 120 por minuto, débil y rítmico, respiraciones 30 por minuto. Se la sondea y se retiran 80 c.c. de orina que se envían al laboratorio. Se procede inmediatamente a la terapéutica de costumbre, a base de bartitúricos, Sulfato de Magnesio, y soluciones dextrosadas al 50\%. Doce horas más tarde la enferma continuaba en el mismo estado de coma, la tensión Mx. y Mn. 0, respiraciones 40 , y anuria. Como el estado de la enferma se hacía más crítico se procede a cambiar la terapéutica y se inicia a las $7 \mathrm{p}$. $\mathrm{m}$. del mismo día el tratamiento con CORTISONA, a razón de $100 \mathrm{mg}$. i.m. cada 8 horas. A las 6 a. m. del día siguiente el estado de coma comienza a ceder, se registra la tensión arterial Mx. 125 y Mn. 80; pulso 100; frecuencia respiratoria 28; y se inicia la diuresis. A las 24 horas de comenzado el tratamiento con dicho medicamento la enferma se encontraba en completo estado de lucidez mental, tranquila, tensión arterial Mx: 160 y Mn: 120, diuresis aumentada. El trabajo de parto se inició a las $5 \mathrm{a}$. $\mathrm{m}$. del día 4 de noviembre y terminó a las 9 y $30 \mathrm{mi}$ nutos p. m., con un niño muerto no macerado y circular del cuello. La dosis total de CORTISONA administrada fue de $500 \mathrm{mg}$.

Exámenes de Laboratorio: Orina, albúmina 3 gms., cilindros hialinos ++ , granulosos +; azohemia $40 \mathrm{mg}$. \%. Kahn negativo.

Caso No 4. Paciente: E. P. de 35 años de edad, de color, multigestante, con embarazo de 8 meses, llega al Servicio de Maternidad el día 17 de febrero casi en estado de anasarca; cefalalgia y epigastralgia; tensión arterial $\mathrm{Mx} .170 \mathrm{y}$ Mn. 90; pulso, frecuencia 90 por minuto; respiraciones 24 ; temperatura $37^{\circ} \mathrm{C}$; ruidos fetales positivos. Se ordenan los exámenes de rutina, reposo absoluto, dieta sin sal, y control de tensión arterial, pulso, y respiración cada 2 horas. Registro de líquido ingerido y eliminado en las 24 horas y se procede a la administración del tratamiento corriente, más Vergytril (Veratrum viride per os), 6 pastillas en las 24 horas. Pese al cuidado diecético y tratamiento rígido, seis días después de su ingreso al Hospital, la enferma es presa de un ataque convulsivo y que se repite una hora más tarde, seguido de un estado de coma. La tensión 
arterial registrada es de 170 de $\mathrm{Mx}$. y 100 de Mn., pulso 110 , respiración estertorosa, 32 por minuto, oliguria marcada, ruidos fetales positivos. Como el estado general de la enferma se hace más grave, se procede a cambiar de terapéutica $y$ se administra CORTISONA a la dosis de $100 \mathrm{mg}$. cada 8 horas intramuscular. A las 24 horas de haber iniciado el tratamiento con dicho medicamento, se observa que el estado comatoso de la enferma comienza a ceder, la tensión arterial registrada es de $160 \mathrm{Mx}$. y $100 \mathrm{Mn}$., pulso 60 por minuto, frecuencia respiratoria 24 , ruidos fetales negativos, duiresis aumentada. El día 23 a la 1 a. m. se inicia el parto y termina a las $3 \mathrm{a}$. m. rápido y espontáneo con niño muerto no macerado. A las 48 horas la enferma recobra su lucidez mental, la diuresis aumenta y la respiración vuelve a su ritmo normal, tensión arterial $160 \mathrm{Mx}$. y $90 \mathrm{Mn}$.; f́recuencia del pulso 64 y rítmico. La paciente se le da la salida el día 6 de marzo del Servicio de Maternidad por curación. La dosis total administrada de CORTISONA fue el 1 gramo.

Exámenes de Laboratorio: Orina, albúmina $5 \mathrm{gm}$.; cilindros hialinos +++ ; granulosos + +; hematíes + +; Glicemia $107 \mathrm{mg}$. \%; Azohemia $24 \mathrm{mg}$. \%; Kahn negativo. Hemograma: 3, 690.000 eritrocitos, leucocitos 12.100; Hemoglobina $75 \%$. Fórmula blanca: polinucleares neutrófilos 88 , eosinófilos y basófilos 0 , linfocitos 12 y monocitos 0 . Fondo de Ojo: ojo derecho, edema papilar, espasmo arterial, con cruces positivas, congestión venosa, pequeñas hemorragias $y$ llamas. Ojo izquierdo, se encontró el mismo cuadro clínico del anterior.

Caso No 5. Paciente: D. N. de 28 años de edad, multigestante, con embarazo gemelar a término, llega al Servicio de Maternidad a las 0-30 minutos del día 5 de abril; tensión arterial: Mx. 120 y Mn. 80; pulso 76; frecuencia respiratoria 24 , y en trabajo franco de parto que se inició el día 4 de abril a las 10 y 30 p. m. y terminó a las 9 a. m. del día siguiente. El primer niño nació a las 8 a. m. espontáneamente y con el segundo hubo necesidad de hacer la extracción podálica una hora más tarde por inercia secundaria de la matriz. Niños en buen estado de salud, y madre aparentemente normal. Siete horas después del nacimiento del último de los niños la enferma es presa de un ataque convulsivo y se procede inmediatamente a la administración del tratamiento de rutina; tres horas más tarde el estado semicomatoso es interrumpido por otro ataque convulsivo, lo que hace cambiar de terapéutica y se procede a la aplicación de CORTISONA a razón de $100 \mathrm{mg}$. cada 8 horas intramuscular. Una hora después la enferma comienza a recobrarse de su estado comatoso y dos horas más tarde la enferma recupera su estado de lucidez mental y entra en estado de franca mejoría. No se volvieron a repetir los ataques convulsivos $y$ antes de las 24 horas del primer ataque la paciente se encontraba completamente restablecida. Se le da salida del Servicio por curación el día 10 de abril. l a dosis total de CORTISONA administrada fue de $500 \mathrm{mg}$.

Exámenes de Laboratorio: Orina, albúmina I gm., Azohemia 28 mg. Gli. cumia $83 \mathrm{mg}$. \% y reacción de Kahn negativo. 

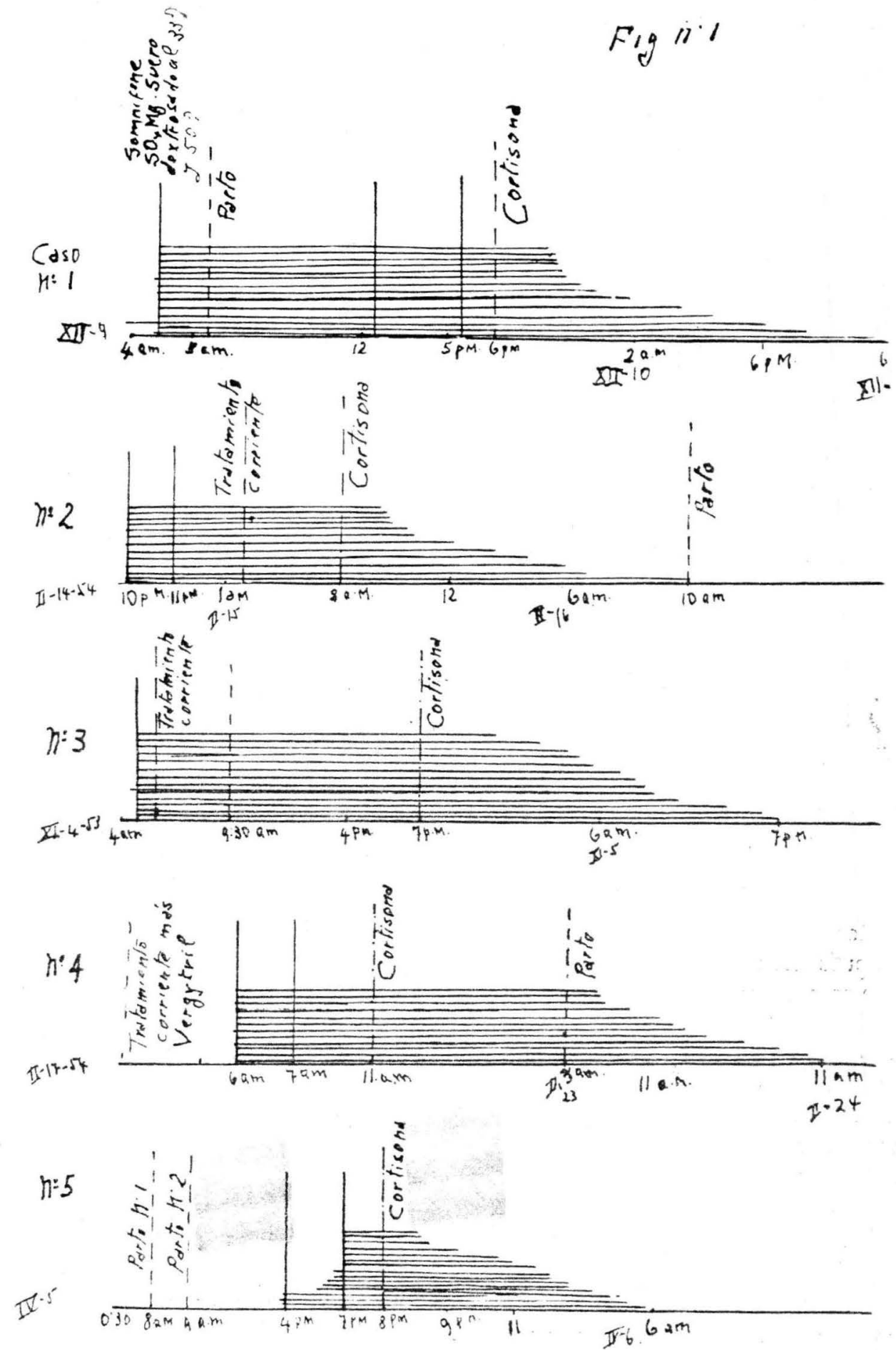

Las line horizutales representin el estado di coma de lit enferma, y las verticales neglas las crisis convulsivas. Los numero representan el tiempo. 
De los casos anteriormente relatados conviene recalcar que en el primero y quinto caso el estado comatoso fue interrumpido por nuevos ataques convul sivos varias heras después del parto, lo que nos demuestra la gravedad de los casos por traterse de eclampsia del puerperio. También es bueno anotar la coincidencia del mejoramiento del estado de coma de todas las enfermas inmediatamente despues de haber comenzado el tratamiento con la CORTISONA.

\section{COMENTARIOS:}

Actualnente es admitido que la corteza suprarrenal ejerce las siguiente acciones maabolicas distintas $\left(^{1}\right)$ :

a) Mantenimiento del balance electrolítico.

b) Infuencia sobre el metabolismo de los hidrocarbonados.

c) Aumento del anabolismo protéico.

Los compuestos más importantes de esta glándula son seis $\left(^{2}\right)$ :

19-Crticosterona.

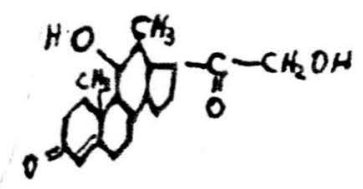

30-17-hidroxi - 11-dehidrocorti costerona. (compuesto $\mathrm{E}$ de Kendall o Cortisona).

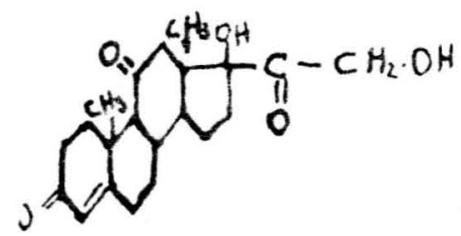

50-11-desoxicorticosterona.

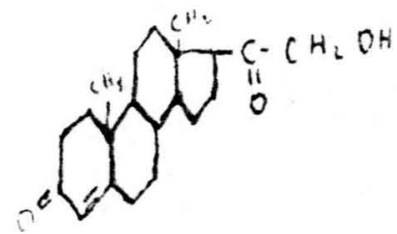

20-11-dehidrocorticosterona.

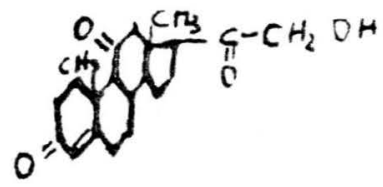

40-17-hidroxicorticosterona

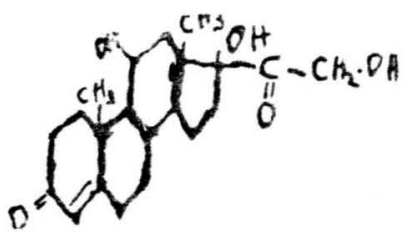

60-17-hidroxi - 11-desoxicorticosterona.

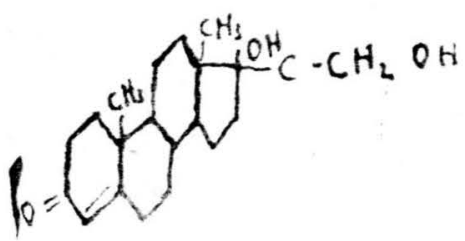


De estos seis compuestos hormonales tenemos: que los cuatro primeros actúan especialmente sobre el metabolismo de los hidrocarbonados, de los cua les la CORTISONA es su integrante más conocido. Las principales caracterís ticas moleculares de tales compuestos son: un 0 con doble ligadura o un $\mathrm{OH}$ con ligadura simple unido al $\mathrm{C}_{11}$; y se les llama hormonas "gluconeogenéticas, glucocorticoides u hormonas S", por promover la deanimación de los aminoácidos y convertirlos en hidratos de carbono, por lo que vienen a estimuar la gluconeogénesis e impedir la desaparición del glucógeno hepático mankniendo los niveles normales de glucosa sanguínea en condiciones de ayuno o cstimulación orgánica.

Los otros dos compuestos, cuyo representante principal es la 11 desoxicorticosterona, están íntimamente relacionados con la regulación del balance electrolítico, de donde el nombre de "mineral-corticoides", se caracterizan por ausencia del grupo $=0 \mathrm{o}-\mathrm{OH}$ unidos al $\mathrm{C}_{11}$, y tienen la propiedad de procucir en el organismo una notable retención de sodio, cloro y agua, aumentande la excre. ción urinaria del fósforo y potasio, sin ejercer efecto alguno sobre el netabolismo de los hidrocarbonados.

De la producción equilibrada de los glucocorticoides y mineral-corticoides depende la defensa general de las reacciones inespecíficas del organisno en el "Síndrome General de Adaptación". Esta respuesta de la corteza suprarenal a las necesidades orgánicas, se cree que tiene lugar a través de la Hipófsis, por producción de corticotrofina y somatotrofina, de las cuales, la primera provoca la descarga de los glucocorticoides, cuyo componente principal es la CORTISONA, y la segunda, los mineral-corticoides. Cuando se produce un de sequilibrio entre éstos, con predominio de la acción de los mineral-corticoiles sobre los glucocorticoides se suceden en el organismo ciertos fenómenos caracerísticos de las "enfermedades de adaptación" cuyos rasgos morfológicos más ípicos son la alteración conectiva llamada "hialinosis", la hipertensión, los ećmas, la albuminuria, la retención del ión sodio, la urea anormal, etc.; síntoms y signos observados en la eclampsia lo que tiende a demostrar que esta enfemedad constituye una enfermedad de adaptación.

Según esta hipótesis se tiene que la eclampsia será producida como conse cuencia de una excesiva producción por parte de la corteza suprarrenal de lo mineral-corticoides con su consecuente disminución de los glucocorticoides, e: decir, que la base hormonal de la eclampsia se debería a un desvío del coeficiente $\frac{\text { glucocorticoides }}{\text { mineral-corticoides }}$ hacia la disminución.

Esta hipótesis se refuerza si tenemos en cuenta que ambos sindromes mejoran con la dieta hiposódica. Así mismo conviene tener presente que tantu la corteza suprarrenal, el riñón y el hígado son los órganos más lesionados en la eclampsia como ha sido comprobado por estudios anatomopatológicos $(:-\mathrm{y}:$ " $)$, órganos cuya integridad anatomica y funcional desempeñan un papel de gran importancia en el síndrome de la intoxicación gravidica. 
En el caso particular de la eclampsia el alarmógeno o "alarming stimulus" de SELYE debe tener su fuente en la placenta, ya que su eliminación es suficiente para que desaparezcan los síntomas. Lo que no se ha dilucidado aún es si los agentes humorales u hormonales actúan simplemente como alérgenos cuya toxicidad no es neutralizada por los glucocorticoides o si la placenta produce en realidad una cantidad excesiva de mineral corticoides o ACTH ( $\left.{ }^{2}\right)$, o cerá la hiperactividad de la corteza suprarrenal el resultado del síndrome de la intoxicación gravídica o la causa de ésta. Son interrogantes por aclarar y que ojalá en un futuro no muy lejano puedan ser resueltos.

En las enfermas eclámpticas se ha observado $\left(2 \mathrm{y}^{5}\right)$ una eliminación subnormal de glucocorticoides en la orina, como también que el pregnandiol y los 17-cctosteroides hállanse disminuídos, y que en cambio las gonadotrofinas están aumentadas. Fauvet y Munzer (") han encontrado en el suero de las eclámpticas niveles bajos de corticotrofina hipofisiaria. Esto nos hace sugerir la hipótesis de que el efecto benéfico de la CORTISONA ${ }^{1}$ en la eclampsia podría deberse a una acción directa, o indirecta bloqueando la secreción de los mineral-corticoides, restableciendo así el equilibrio hormonal perdido y produciendo a su vez como consecuencia la restauración del síndrome general de adaptación, lo cual hace ver su significación y resultados prácticos.

\section{RESUMENES Y CONCLUSIONES:}

10--Son tratados cinco casos de coma ecláptico con CORTISONA y se obtuvo en todos ellos la curación.

$2^{\circ}$ - La dosis total fue de $500 \mathrm{mg}$. en cuatro de las observaciones presentadas y de $1 \mathrm{gm}$. en una sola de ellas.

30-En todas las enfermas se observó una notoria mejoría de los edemas, estado de coma, diuresis, cesación de los ataques, convulsiones, sedación mental; inmediatamente después de la administración de dicho medicamento.

$4^{\circ}$ - En tres de los casos la muerte del feto, sobrevino antes de la aplicación de la CORTISONA, y en uno de los casos se produjo dos horas después de la administración de ésta.

50-En ninguna de las enfermas se produjo elevación de la tensión arterial sobre la registrada antes de la administración de la CORTISONA. Por el contrario, se observó un descenso de la máxima en 3 de los casos.

$6^{\circ}$-Las observaciones que aquí se presentan, sumadas a las registradas en la bibliografía médica extranjera, pueden servir de base para intentar una revisión de los tratamientos médicos actuales de la Eclampsia.

79-La hipotesis de las enfermedades de adaptación parece darnos un poco de luz sobre la etiopatogenia de la Eclampsia y su respectivo tratamiento.

80-De las cinco enfermas dos eran primigestantes y tres multigestantes. Dos eclampsias del puerperio y tres del parto. 


\section{B I B L I O G R A F I A}

1.-Wilkins L.: "Diagnóstico y tratamiento de los trastornos endocrinos en la infancia y adolescencia”. Editorial Médico Quirúrgica. 1953.

2.-Pascualini R. Q.: "Stress. Enfermedades de adaptación A.C.T.H. y Cortisona". Editorial "El Ateneo". 1952.

3.-Pérez M. L.: "Tratado de Obstetricia". Aniceto López editor. Tomo No 2.

4.-Merck y Co. (Editor): "The Cortisone investigator". (1950, 1951), Rahway, N. J.

5.-De Feo D. A. y Nusimovich B.: "Eclampsia tratada con Cortisona". Semana Médica. 99: 179; 1951.

6.-Mendive J. R.: "Química y farmacología de la corticotrofina y cortisona”. Semana Médica, 99: 913; 1951.

7.-Barnes A. C. Collective Review: "A.C.T.H. and Cortisone in Obstetrics and Gynecology". 3: 322; 1954.

8.-Venning E. H., Ph. D., Singer B. Ph. D., Simpson G. A.: "Adrenocortical Function in Toxemia of Pregnancy". 67: 542; 1954.

9.-Moore J., Jessop W. J. E., O'Donovan, D. K. Barry, A. P., Quinn B., Brit. M. J. 1: 841-850; 1951.

10.-Silva-Mojica C. R.: "Observaciones sobre los tratamientos de la Eclampsia". Rev Col. de Obstetricia y Ginecología. 5: No 3, 159; 1953-54.

11.-Dexeus Font S.: "Tratado de Obstetricia". Salvat. 2: 625-636; 1949.

12.-Nubiola-Zarate: Editorial Labor, S. A. 2: 700-720; 1951.

13.-Mastboom J. L.: "Importance of Adrenocortical Steroids for Development of Toxemia of Late Pregnancy", Year Book of Obstetrics and Gynecoloy. Pag. 79. 1953-54. 